\title{
Frequency of narcissistic personality disorder in a counseling center population in China
}

XueFeng Jiang ${ }^{1}$, JunJie Wang ${ }^{1,2+}$, Wei Sun ${ }^{3+}$, LiHua Xu ${ }^{1}$, XiaoChen Tang ${ }^{1}$, HuiRu Cui ${ }^{1}$, YanYan Wei ${ }^{1}$, Li Hui ${ }^{2}$, Yi Qiao $^{1 *}$, JiJun Wang ${ }^{1,4,5^{*}}$ and TianHong Zhang ${ }^{1 *}$

\begin{abstract}
Background: Narcissistic personality disorder (NPD) has never been applied in Chinese clinical practice, and the distribution of NPD in the clinical population of China is largely unknown. The current study uses two-stage clinicbased screening to investigate the frequency and clinical features of NPD in a Chinese help-seeking sample.

Methods: A total of 1402 consecutive outpatients ages 18-60 were recruited during their visit to the Shanghai Mental Health Center and screened with the Personality Diagnostic Questionnaire Fourth Edition Plus (PDQ-4+) and Structured Clinical Interview for the Diagnostic and Statistical Manual of Mental Disorders (DSM-IV) Axis II (SCID-II). The structured clinical interview was administered to estimate the rate of NPD and the frequency of each disorder criterion.

Results: The frequency estimate of NPD in the total sample was 4.0\%. Among the 56 outpatients who met the criteria for NPD, there were more males than females, and many had a better educational background. The SCID-II interviews revealed high frequencies of diagnostic criterion 1 ("exaggerated sense of self-importance. NPD likely overlaps with Histrionic PD, Borderline PD, and Paranoid PD. This two stage screening method can enhance detection of Chinese NPD patients in clinical settings.

Conclusions: Narcissism pathology is not rare in the Chinese psychiatric community when using the DSM-IV NPD criteria. Existing evidence suggests, at least indirectly, that there are important benefits of NPD diagnosis in psychiatric practice.
\end{abstract}

Keywords: Narcissism, DSM, Diagnosis, Narcissistic personality disorder, China

\section{Background}

Narcissistic Personality Disorder (NPD) was first recognized in the Diagnostic and Statistical Manual of Mental Disorders, third edition (DSM-III) [1] in 1980. NPD patients are featured as having an exaggerated (fantastic or behavioral) behavior pattern requiring praise and lacking empathy. The disorder is widely used in clinical practice and widely accepted as an abnormal personality characteristic in North America [2, 3]. In the Chinese

\footnotetext{
* Correspondence: qiaoyi2004@msn.com; jijunwang27@163.com; zhang_tianhong@126.com

JunJie Wang and Wei Sun share first authorship.

${ }^{1}$ Shanghai Mental Health Center, Shanghai Jiaotong University School of Medicine, Shanghai Key Laboratory of Psychotic Disorders (No.13dz2260500), 600 Wanping Nan Road, Shanghai 200030, People's Republic of China Full list of author information is available at the end of the article
}

Classification and Diagnosis of Mental Diseases, Third Edition (CCMD-3) [4] in 2001, NPD was classified as a category of other or unspecific personality disorders [F60.8-F60.9]. Although NPD has not been included in the 11th revision of the International Classifications of Diseases and Related Health Problems (ICD-11), a large number of studies in North America in the past 30 years have demonstrated the reliability of this diagnostic validity and its independence as a diagnostic category. Therefore, the diagnostic criteria for NPD have been retained from the DSM-III to the DSM-5 [5] and have been revised to varying degrees. Notably, the "alternative model" described in the DSM-5 has curtailed the PD categories to six specific PDs [6], which include NPD.

(c) The Author(s). 2019 Open Access This article is distributed under the terms of the Creative Commons Attribution 4.0 International License (http://creativecommons.org/licenses/by/4.0/), which permits unrestricted use, distribution, and reproduction in any medium, provided you give appropriate credit to the original author(s) and the source, provide a link to the Creative Commons license, and indicate if changes were made. The Creative Commons Public Domain Dedication waiver (http://creativecommons.org/publicdomain/zero/1.0/) applies to the data made available in this article, unless otherwise stated. 
Although the clinical features of NPD are distinct, the distribution of NPD in the Chinese clinical population remains largely unknown due to lack of appropriate diagnostic classification in Chinese clinical practice [7]. Moreover, the high rates of overlap between NPD and other types of PDs found in previous studies $[3,8]$, make it more difficult to generalize NPD in China [9]. However, narcissism is a very important issue in the theoretical study of psychology and psychotherapy. In clinical practice, effective identification of NPD is not only important for the diagnosis of mental disorders and differential diagnosis, but is also directly related to the formulation of treatment strategies. To be specific, affective relevant symptoms in NPD patients may be easier identified and this makes the antidepressant effects become the only target of treatment. We speculate that make a diagnosis of NPD may represent a new target for the development of effective therapeutic strategies (such as psychoanalysis [10]) to guide systematic analysis of the grandiose self. This diagnosis process also leads to a shift from symptom-centered treatment to personality rehabilitation, by the gradual integration of a grandiose, split-off self into a more integrated stable concept of the self. [11] This study is an exploratory study on the preliminary clinical application of NPD in China. The distribution of NPD, clinical and demographic characteristics, will be investigated in order to discuss the feasibility of the application of NPD diagnostic criteria in China.

\section{Methods}

\section{Subjects and procedures}

This study was approved by the Research Ethics Committee of the Shanghai Mental Health Center (SMHC) and conducted following the tenets of the Helsinki Declaration. Every 10th outpatient in the psychocounseling clinics was selected and a total of 1402 consecutive outpatients were recruited. All the participants gave written informed consent at the recruitment stage of the study. Study inclusion criteria were: between 18 and 60 years of age and with an educational background of at least junior middle school. Exclusion criteria were: outpatients with acute attacks of psychoses, severe somatic diseases, and diagnoses of mental retardation or dementia. Details of the study procedures can be found elsewhere [7, 12-15].

Outpatients were screened using the Personality Diagnostic Questionnaire Fourth Edition Plus (PDQ$4+)$ [16]. Positive screening was defined as a total score of 29 or higher on the PDQ-4+, or specific PD subscale scores of $>4$ or 5 . Outpatients whose PDQ$4+$ test results were positive referred to two senior psychiatrists (5 years' experience of psychiatry), each of whom received 2 weeks of training to perform the Structured Clinical Interview for DSM-IV Axis II (SCID-II). NPD diagnosis was confirmed using the questionnaire and face-to-face interview with a twostage process.

\section{Measures}

PDs were assessed with the PDQ-4+ and SCID-II. Our previous study [7] and other research has validated that the PDQ-4+ has a high sensitivity (0.89) and moderate specificity (0.65) for PD screening. The SCID-II [17] was designed to assess the DSM-IV 10 PDs. Our team translated and implemented the Chinese version of the SCIDII. Besides, previous reports have demonstrated that the Chinese version SCID-II has a median coefficient for internal consistency of 0.70 , with a relatively high test-retest reliability (0.70). In the DSM-IV, there are nine diagnostic criteria for NPD. The PDQ-4+ and SCID-II were derived from DSM-IV and have a one-to-one relationship with it. Therefore, they can better reflect the diagnostic criteria for NPD in the DSM-IV. The corresponding relationship between the PDQ-4+, SCID-II, and the DSM-IV criteria can be found in Table 1.

\section{Statistical analysis}

To explore whether demographic variables were risk factors for the NPD group, the frequency rates and odds ratios (OR) were examined, and 95\% confidence intervals (CIs) were estimated. Group comparisons of demographic and clinical variables were evaluated using Pearson Chin-Square tests for categorical variables and two-tailed tests for continuous variables. Significance was reached when $p<0.05$. We presented the positive rate of each NPD diagnostic criteria (DSM-IV) identified by the PDQ-4+ and SCID-II. Finally, binary logistic regression analysis was used to explore independent predictive factors for the outpatients who were diagnosed or comorbid with DSM-IV NPD and calculate OR with $p$-values and 95\% CIs.

\section{Results}

In this study, 1511 outpatients were randomly selected from the psychological counseling department. Among them, $1402(92.8 \%)$ were willing to complete the study. Among the 1402 subjects, the proportion of women $(n=761,54.3 \%)$ was slightly higher than that of men $(n=641,45.7 \%)$. The average age was $30.5 \pm 9.6$ years old. There were $458(32.7 \%)$ aged $18-24$ years old, 533 (38.0\%) aged 25-34 years, 263 (18.8\%) aged 35-44 years, and the remaining 148 people (10.5\%) were aged over 45 years old. The majority sample of the current study was younger than 35 years old (70.7\%). There was no significant difference in age between the male and female groups $(t=1.55, p>0.05)$. There were $986(70.3 \%)$ 
Table 1 Correspondence between DSM-IV diagnostic criteria for narcissistic personality disorder (NPD) in PDQ-4+ and SCID-II

\begin{tabular}{lll}
\hline Diagnostic Criteria & Screen & Interview \\
(DSM-IV, NPD) & PDQ $^{4+}(\mathrm{NPD})$ & SCID-II (NPD)
\end{tabular}

A long-term pattern of abnormal behavior characterized by exaggerated feelings of self-importance, an excessive need for admiration, and a lack of empathy. The disorder begins by early adulthood and is indicated by at least five of the following:

(1) An exaggerated sense of self-importance (e.g., I have achieved much more than others exaggerates achievements and talents, expects think. (Item-5) to be recognized as superior without commensurate achievements)

(2) Preoccupation with fantasies of unlimited success, power, brilliance, beauty, or ideal love

(3) Believes he is "special" and can only be understood by, or should associate with, other special or high-status people (or institutions)

(4) Requires excessive admiration

(5) Has a sense of entitlement

(6) Selfishly takes advantage of others to achieve his own ends

Some people think I use others. (Item-68).

I need people to pay attention to me or to praise me. (Item-44)

When a salesperson makes me wait in front of the counter or wait for a long time, I tend to feel indignant and angry. (Item-57)

Most people do not appreciate your extraordinary talents or achievements. (Item-73).

Some people say that you have too high self-evaluation. (Item-74),

You say you often think that 1 day you will gain power, reputation or praise. (Item-75).

You say you often think that 1 day you will have a perfect love story. (Item-76).

When you encounter problems, you almost always insist on meeting senior leaders. (Item-77).

You think it's only worth spending time with special or important people. (Item-78).

People pay attention to or envy you in some way, which is very important to you. (Item-79).

When certain rules or social conventions interfere with you, you do not think it is necessary to comply with them. (Item-80).

You often feel that others are giving you special treatment. (Item-81)

You often feel that it is necessary to offend some people in order to get what you want. (Item-82).

You often have to put your needs above others. (Item-83).

You often want others to look at your face and do what you want without doubt. (Item-84).

(7) Lacks empathy

People often blame me for not realizing that they are in a bad mood. (Item-73).

You often think that it doesn't matter how to treat others' thoughts or feelings. (Item-85).

Some people are jealous of me. (Item-79).

(8) Is often envious of others or believes that others are envious of him

(9) Shows arrogant, haughty, patronizing, or Others think I'm arrogant. (Item-92).
When others do well, you will be angry. (Item-86).

You feel that people are often jealous of you. (Item-87).

You think that few people are worth your time or attention. (Item-88).

Note: PDQ-4+: The Personality Diagnostic Questionnaire Version 4 plus. SCID-II: Structured Clinical Interview for DSM-IV Axis II

screened positive for PD by the PDQ-4+. A total of 539 (38.4\%) patients were diagnosed with PD. The frequency of PD was $38.4 \%$ (95\% CI, 35.9-40.9\%). In addition, the patients with PD were younger, more likely to have single marital statuses, and had fewer years of education than those without PD.

There were 56 patients who met the criteria for NPD, with a frequency rate of $4.0 \%$ (Table 2). The frequency of NPD was higher in males than in females $(\mathrm{OR}=4.9$, 95\% CI: 2.5-9.3), by about a factor of five. NPD patients completed more years of education $(\mathrm{OR}=0.4,95 \% \mathrm{CI}$ : $0.2-0.8)$ than other patients.

The positive rate of each diagnostic criterion of NPD in the self-report questionnaire and structured interview in the overall sample is listed in Table 3. The diagnostic criterion 4 (requiring excessive envy) had the highest positive rate in both the self-report and interview, followed by the self-rated subjects who considered themselves "special" and empowered (criterion 3, 5). In addition, in gender comparisons, except for criteria 4 and 9, there were more males than females who reported NPD symptoms, especially for criterion 1 (an exaggerated sense of self-importance).

To investigate the specificity of the NPD diagnostic criteria, we explored the positive rates of NPD criteria in other types of PDs (Table 4). NPD is basically different from other types of PDs (positive rate is higher than other types), but some diagnostic items have high positive rates in other PDs. For example, for criterion 4 (needs too much envy), the positive rate in Histrionic PD is as high as $60.9 \%$, which is close to $69.6 \%$ of NPD's positive rate. In addition, according to the positive rate 
Table 2 Prevalence and demographics of narcissistic personality disorder (NPD) in Chinese psychological counseling outpatients

\begin{tabular}{|c|c|c|c|c|c|c|c|}
\hline & In total & $\mathrm{NPD}(\mathrm{n})$ & $(\%)$ & $95 \% \mathrm{Cl}$ & Comparison & $O R$ & $95 \% \mathrm{Cl}$ \\
\hline Sample in all & 1402 & 56 & 4.0 & $2.97 \% \sim 5.03 \%$ & - & - & - \\
\hline \multicolumn{8}{|l|}{ Gender } \\
\hline 1. Male & 641 & 45 & 7.0 & $5.04 \% \sim 9.00 \%$ & \multirow[t]{2}{*}{$1 \sim 2$} & \multirow[t]{2}{*}{4.857} & \multirow[t]{2}{*}{$2.533 \sim 9.311$} \\
\hline 2. Female & 761 & 11 & 1.4 & $0.60 \% \sim 2.29 \%$ & & & \\
\hline \multicolumn{8}{|l|}{ Age } \\
\hline 1. 18-28(years) & 730 & 30 & 4.1 & $2.67 \% \sim 5.55 \%$ & \multirow[t]{2}{*}{$1 \sim 2$} & \multirow[t]{2}{*}{1.062} & \multirow[t]{2}{*}{$0.635 \sim 1.777$} \\
\hline 2. 29-60(years) & 672 & 26 & 3.9 & $2.41 \% \sim 5.33 \%$ & & & \\
\hline \multicolumn{8}{|l|}{ Educational background } \\
\hline 1. High school degree or below & 596 & 13 & 2.2 & $1.01 \% \sim 3.35 \%$ & \multirow[t]{2}{*}{$1 \sim 2$} & \multirow[t]{2}{*}{0.409} & \multirow[t]{2}{*}{$0.222 \sim 0.753$} \\
\hline 2. College degree or above & 806 & 43 & 5.3 & $3.78 \% \sim 6.89 \%$ & & & \\
\hline \multicolumn{8}{|l|}{ Personal income (/ month / RMB) } \\
\hline 1. $\sim 1000$ & 518 & 22 & 4.2 & $2.51 \% \sim 5.98 \%$ & $1 \sim 2$ & 1.499 & $0.776 \sim 2.896$ \\
\hline 2. $1000 \sim 3000$ & 494 & 14 & 2.8 & $1.37 \%$ 4.30\% & $1 \sim 3$ & 0.828 & $0.459 \sim 1.496$ \\
\hline 3. 3000 & 390 & 20 & 5.1 & $2.94 \% \sim 7.32 \%$ & $2 \sim 3$ & 0.553 & $0.283 \sim 1.080$ \\
\hline
\end{tabular}

Note: $95 \%$ Cl 95\% confidence interval, OR odds ratio

of NPD patients, the importance of self-exaggeration (Criterion 1) is the highest common symptom, followed by Criterion 2 (73.2\%).

To identify risk factors for NPD diagnoses, logistic regression (forward stepwise) analyses were performed. The presence of NPD was applied as the dependent variable, while demographic characteristics and other PD diagnoses were listed as independent variables. As shown in Table 5, demographic factors, such as male gender, an older age, and a higher educational level, were associated with the diagnosis of NPD. Patients having other PDs, such as Histrionic PD, Borderline PD, Paranoid PD, and Schizotypal PD, was a significant predictor of meeting criteria for NPD diagnosis. Those patients with Avoidant PD were less likely to be diagnosed with NPD. The strongest effect was derived from gender.

\section{Discussion}

The diagnostic criteria for NPD have been changing and developing since NPD was officially listed in the DSMIII in 1980. At the same time, the debate over the validity and reliability of NPD has never stopped [6, 18]. Because of these controversies, NPD has rarely been used in clinical practice of psychiatry in China, and therefore the frequency of NPD in the Chinese clinical population is largely unknown. Otherwise, it has been reported that the frequency rate of NPD has been increasing recently in other countries $[8,19,20]$. To our knowledge, our research group is the first to investigate the distribution of the NPD outpatients in an epidemiological way in a clinical setting in mainland China. The frequency of NPD in an outpatient counseling setting was $4.0 \%$. In gender comparison, we believe that there are differences

Table 3 Positive rate of each NPD diagnostic criteria (DSM-IV) identified by PDQ-4+ and SCID-II, and their sex difference

\begin{tabular}{|c|c|c|c|c|c|c|c|c|c|c|c|}
\hline \multirow{3}{*}{$\begin{array}{l}\text { Diagnostic } \\
\text { Criteria }\end{array}$} & \multirow{2}{*}{\multicolumn{3}{|c|}{$\begin{array}{l}\text { Screened Positively by } \\
\operatorname{PDQ}^{4+}(N=1402 \text { in total })\end{array}$}} & \multicolumn{8}{|c|}{ Interviewed Positively by SCID-II ( $N=986$ in total) } \\
\hline & & & & \multicolumn{2}{|c|}{ In total PD sample } & \multicolumn{2}{|c|}{ Male $(N=460)$} & \multicolumn{2}{|c|}{ Female $(N=526)$} & \multicolumn{2}{|c|}{$\begin{array}{l}\text { Male vs. Female } \\
\text { comparison }\end{array}$} \\
\hline & $n$ & Positive rate(\%) & $95 \% \mathrm{Cl}$ & $\mathrm{n}$ & Positive rate(\%) & $n$ & Positive rate(\%) & $\mathrm{n}$ & Positive rate(\%) & $x^{2}$ & $P$ \\
\hline 1 & 552 & 39.4 & $34.6-44.3$ & 164 & 16.6 & 104 & 22.6 & 60 & 11.4 & 22.207 & $0.000^{* * *}$ \\
\hline 2 & 612 & 43.7 & $38.8-48.6$ & 223 & 22.6 & 130 & 28.3 & 93 & 17.7 & 15.696 & $0.000^{* * *}$ \\
\hline 3 & 647 & 46.1 & $41.2-51.1$ & 98 & 9.9 & 64 & 13.9 & 34 & 6.5 & 15.212 & $0.000^{* * *}$ \\
\hline 4 & 762 & 54.4 & $49.5-59.3$ & 233 & 23.6 & 117 & 25.4 & 116 & 22.1 & 1.555 & 0.212 \\
\hline 5 & 634 & 45.2 & $40.3-50.1$ & 180 & 18.3 & 100 & 21.7 & 80 & 15.2 & 7.012 & $0.008^{* *}$ \\
\hline 6 & 239 & 17.0 & $13.3-20.7$ & 224 & 22.7 & 119 & 25.9 & 105 & 20.0 & 4.878 & $0.027^{*}$ \\
\hline 7 & 278 & 19.8 & $15.9-23.8$ & 79 & 8.0 & 48 & 10.4 & 31 & 5.9 & 6.867 & $0.009^{* *}$ \\
\hline 8 & 520 & 37.1 & $32.3-41.9$ & 97 & 9.8 & 55 & 12.0 & 42 & 8.0 & 4.364 & $0.037^{* *}$ \\
\hline 9 & 428 & 30.5 & $25.9-35.1$ & 117 & 11.9 & 63 & 13.7 & 54 & 10.3 & 2.760 & 0.097 \\
\hline
\end{tabular}


Table 4 Positive rate (\%) of each NPD diagnostic criteria (DSM-IV) among specific PD

\begin{tabular}{|c|c|c|c|c|c|c|c|c|c|c|c|c|}
\hline Diagnostic Criteria & NPD & HIS & BOR & ANT & PAR & $\mathrm{SCH}$ & SCHT & AVO & DEP & $\mathrm{OBC}$ & DPS & PAS \\
\hline & $n=56$ & $n=46$ & $n=98$ & $n=16$ & $n=71$ & $n=66$ & $n=74$ & $n=146$ & $n=40$ & $n=121$ & $n=72$ & $n=57$ \\
\hline 1 & 82.1 & 26.1 & 16.3 & 31.3 & 32.4 & 12.1 & 27.0 & 10.3 & 2.5 & 26.4 & 5.6 & 35.1 \\
\hline 2 & 73.2 & 37.0 & 34.7 & 12.5 & 39.4 & 15.2 & 37.8 & 21.9 & 17.5 & 29.8 & 20.8 & 28.1 \\
\hline 3 & 58.9 & 15.2 & 14.3 & 18.8 & 22.5 & 4.5 & 16.2 & 6.2 & 2.5 & 16.5 & 4.2 & 12.3 \\
\hline 4 & 69.6 & 60.9 & 29.6 & 25.0 & 29.6 & 7.6 & 29.7 & 15.8 & 27.5 & 31.4 & 16.7 & 33.3 \\
\hline 5 & 66.1 & 15.2 & 23.5 & 25.0 & 33.8 & 24.2 & 23.0 & 14.4 & 15.0 & 23.1 & 13.9 & 36.8 \\
\hline 6 & 67.9 & 30.4 & 37.8 & 25.0 & 40.8 & 21.2 & 33.8 & 19.2 & 25.0 & 25.6 & 29.2 & 42.1 \\
\hline 7 & 50.0 & 6.5 & 9.2 & 25.0 & 12.7 & 18.2 & 8.1 & 8.9 & 7.5 & 11.6 & 5.6 & 21.1 \\
\hline 8 & 58.9 & 19.6 & 16.3 & 18.8 & 18.3 & 7.6 & 17.6 & 8.2 & 7.5 & 22.3 & 6.9 & 17.5 \\
\hline 9 & 46.4 & 13.0 & 15.3 & 12.5 & 18.3 & 21.2 & 17.6 & 11.6 & 7.5 & 15.7 & 13.9 & 15.8 \\
\hline
\end{tabular}

Note: Cluster A PD: Paranoid PD (PAR); Schizoid PD (SCH); Schizotypal PD (SCHT); Cluster B PD: Histrionic PD (HIS); Narcissistic PD (NAR); Borderline PD (BOR); Antisocial PD (ANT); Cluster C PD: Avoidant PD (AVO); Dependent PD (DEP); Obsessive-compulsive PD (OBC); In the appendix of DSM-IV: Depressive PD (DPS);Passive-aggressive PD (PAS)

between male and female NPD, male frequency is higher than female frequency, which is consistent with Torgersen's research [21]. Furthermore, NPD patients characterized as having a higher education level than others, suggesting that NPD patients might be used to be high function. These exploratory data will help Chinese psychiatrists reconsider the applicability of NPD in the Chinese population.

The co-morbidity with other types of PDs is also one of the main problems hindering the application of NPD in China. In this study, we found that NPD likely overlaps with Histrionic PD and Borderline PD, which are both in cluster B, whereas Paranoid PD is in cluster A. These three types of PD also entered the logistic regression model at the same time, indicating that patients with any of these three PDs are more likely to meet NPD criteria as well. Clinical discrimination is needed to compare NPD with these types of PDs.

From 1914, when Freud published his dissertation, "On Narcissism," to 1931, when Freud formally proposed narcissistic personality types, NPD diagnosis has been growing. With the continuous development of narcissism theory since 1980, NPD diagnostic criteria have entered continuous revision in the DSM diagnostic system, and the whole process has spanned almost a century. The current diagnostic criteria of NPD in the DSM-IV can be clearly compared with the symptoms of patients in clinical application, and many experimental studies have proved that NPD can be used as an independent diagnostic criterion [22, 23]. However, this historical background of the completely western culturebased diagnosis criteria of NPD may cause overlap and be confused with other types of PDs when NPD applied in countries with different cultural backgrounds [24]. Future research should attempt to answer the question as to what these cultural differences are. This then leads to the question as to how to modify the NPD criteria to fit in a Chinese cultural context.

Consistently, all criteria for NPD in the DSM-IV were arranged in descending order of importance [25]. This order has also been confirmed to some extent in this study. The order of the positive rate of NPD criteria is 1 , 2, 4, 6, 5, 3, 8, 7, 9 (criterion number). However, we also found those NPD diagnostic criteria are common in other types of PDs in Cluster B. This is also confirmed by Morey et al. [26]. The co-morbidity rate of NPD and Histrionic PD is as high as $53.1 \%$ followed by Borderline PD (46.9\%). It has been suggested that the clinical

Table 5 Logistic regression for risk factors predicting the diagnosis of Narcissistic PD

\begin{tabular}{|c|c|c|c|c|c|c|c|}
\hline Variable & Beta & SE & Wald statistic & $P$ value & Odds ratio & $95 \% \mathrm{Cl}$ & \\
\hline Gender & 2.774 & 0.467 & 35.298 & 0.000 & 16.025 & 6.417 & 1.106 \\
\hline Age (years) & 0.065 & 0.018 & 12.840 & 0.000 & 1.067 & 1.030 & 40.016 \\
\hline Educational Level & 1.541 & 0.386 & 15.897 & 0.000 & 4.669 & 2.189 & 9.960 \\
\hline Paranoid PD & 1.390 & 0.440 & 9.987 & 0.002 & 4.016 & 1.696 & 9.511 \\
\hline Schizotypal PD & 1.107 & 0.542 & 4.175 & 0.041 & 3.025 & 1.046 & 8.744 \\
\hline Histrionic PD & 2.233 & 0.539 & 17.131 & 0.000 & 9.326 & 3.240 & 26.845 \\
\hline Borderline PD & 1.727 & 0.504 & 11.729 & 0.001 & 5.625 & 2.093 & 15.115 \\
\hline Avoidant PD & -2.473 & 1.053 & 5.514 & 0.019 & 0.084 & 0.011 & 0.664 \\
\hline
\end{tabular}


differentiation of other types of Cluster B PDs should be strengthened when NPD diagnostic criteria are applied in China. The current NPD diagnosis is a categorical approach which has been questioned for its arbitrary diagnostic thresholds and extensive overlap with other PD categories in DSM-IV. The current global trend is that the categorical PD diagnoses transfer to the dimensional-categorical (DSM-5) and dimensional (ICD11) approaches. Further studies should also examine whether dimensional approaches can improve the validation of NPD diagnosis in Chinese population.

Our study has several limitations, including that the study was designed as a cross-sectional, open and singlecenter study. The data may not be representative of the entire Chinese population since recruitment were conducted only at a single site. However, the SMHC is the largest psychiatric service center in China (serving over 800,000 outpatients per year) and provides professional treatment for patients throughout the country, and about half of the current sample were not Shanghai natives. However, although a single-site design may increase sample homogeneity and continuity, it also could limit the generalisability of the findings. The SCID-II clinical interview was only conducted for those whose self-reported PDQ-4+ screening was positive that could result in underestimates of the frequency of NPD by the exclusion of participants who were screened negative. Finally, our sample is recruited from outpatients, the possibility of clinical state effects may impact our PD assessment.

\section{Conclusions}

In summary, although the diagnosis of DSM-5 NPD is not included in Chinese psychiatric practice, our results suggest that NPD is not rare in psychiatric outpatients in China. Further longitudinal studies should examine the validity and reliability of NPD diagnosis among Chinese patients using a prospective design.

\section{Abbreviations}

CCMD-3: Chinese Classification and Diagnosis of Mental Diseases, Third Edition; Cl: confidence interval; DSM-III: Diagnostic and Statistical Manual of Mental Disorders, third edition; ICD-11: The 11th revision of the International Classifications of Diseases and Related Health Problems; NPD: Narcissistic Personality Disorder; OR: Odds ratios; PDQ-4 + : Personality Diagnostic Questionnaire Fourth Edition Plus; SCID-II: Structured Clinical Interview for DSM-IV Axis II

\section{Publisher's Note}

Springer Nature remains neutral with regard to jurisdictional claims in published maps and institutional affiliations.

\section{Acknowledgements}

None.

\section{Authors' contributions}

$\mathrm{THZ}$ and XFJ conceptualized the study, wrote the first draft of manuscript and conducted the statistical analyses. JJW1 and WS helped in the design of the study and edited the manuscript. LHX and XCT interviewed participants and collected and organized the primary data. HRC and YYW managed the literature searches, statistical analyses and edited the manuscript. LH, YQ and JJW2 designed the study and provided supervision in the implementation of the study. All authors have approved the final manuscript.

\section{Funding}

This study was supported for this subsequent statistical analysis were provided in part by Ministry of Science and Technology of China, National Key R\&D Program of China (2016YFC1306803, 2016YFC1306805), National Natural Science Foundation of China (81671329, 81671332), Suzhou Municipal Science and Technology Bureau, China (sys2018097), Clinical Research Plan of SHDC (16CR2015A, 16CR3016A), Science and Technology Commission of Shanghai Municipality (19441907800), Shanghai Jiaotong University Foundation (ZH2018ZDB03).

This study had significant institutional support in Shanghai Mental Health Center by The Clinical Research Center at Shanghai Mental Health Center (CRC2018ZD01, CRC2018ZD04 and CRC2018YB01), Shanghai Key Laboratory of Psychotic Disorders (13dz2260500), Shanghai Mental Health Center Foundation (2016-FX-01, 2017-TSXK-03), and Shanghai Key Basic Research Project (16JC1420201).

\section{Availability of data and materials}

The dataset(s) generated during the current study are not publically available due to ethical restrictions but are available from the corresponding author on reasonable request.

\section{Ethics approval and consent to participate}

This study was conducted following the tenets of the Helsinki Declaration and approved by the Research Ethics Committee of the Shanghai Mental Health Center (SMHC). All participants provided written informed consent to participate in this study.

\section{Consent for publication}

Not applicable.

\section{Competing interests}

Author TianHong Zhang is currently acting as an Associate Editor for BMC Psychiatry. The authors declare that they have no competing interests.

\section{Author details \\ ${ }^{1}$ Shanghai Mental Health Center, Shanghai Jiaotong University School of Medicine, Shanghai Key Laboratory of Psychotic Disorders (No.13dz2260500), 600 Wanping Nan Road, Shanghai 200030, People's Republic of China. ${ }^{2}$ Institute of Mental Health, Suzhou Guangji Hospital, The Affiliated Guangji Hospital of Soochow University, Soochow University, Suzhou 215137, Jiangsu, China. ${ }^{3}$ Department of Neurosurgery, Pu Nan Hospital, Shanghai 200125, China. ${ }^{4}$ CAS Center for Excellence in Brain Science and Intelligence Technology (CEBSIT), Chinese Academy of Science, Shanghai, People's Republic of China. ${ }^{5}$ Bio-X Institutes, Key Laboratory for the Genetics of Developmental and Neuropsychiatric Disorders (Ministry of Education), 600 Wanping Nan Road, Shanghai 200030, People's Republic of China.}

Received: 17 January 2019 Accepted: 17 June 2019

Published online: 05 July 2019

References

1. American Psychiatric Association. Diagnostic and statistical manual of mental disorders. 3rd ed. Washington: American Psychiatric Association; 1980.

2. Klein DN, Riso LP, Donaldson SK, Schwartz JE, Anderson RL, Ouimette PC, Lizardi $\mathrm{H}$, Aronson TA. Family study of early-onset dysthymia. Mood and personality disorders in relatives of outpatients with dysthymia and episodic major depression and normal controls. Arch Gen Psychiatry. 1995;52(6):487-96.

3. Stinson FS, Dawson DA, Goldstein RB, Chou SP, Huang B, Smith SM, Ruan WJ, Pulay AJ, Saha TD, Pickering RP, et al. Prevalence, correlates, disability, and comorbidity of DSM-IV narcissistic personality disorder: results from the wave 2 national epidemiologic survey on alcohol and related conditions. J Clin Psychiatr. 2008;69(7):1033-45.

4. Chinese Society of Psychiatry. The Chinese classification and diagnostic criteria of mental disorders version 3 (CCMD-3). Jinan: Chinese Society of Psychiatry; 2001. 
5. American Psychiatric Association. Diagnostic and statistical manual of mental disorders. 5th ed. Washington: American Psychiatric Association; 2013.

6. Oldham JM. The alternative DSM-5 model for personality disorders. World Psychiatr. 2015;14(2):234-6.

7. Zhang T, Wang L, Good MJ, Good BJ, Chow A, Dai Y, Yu J, Zhang H, Xiao Z. Prevalence of personality disorders using two diagnostic systems in psychiatric outpatients in Shanghai, China: a comparison of uni-axial and multi-axial formulation. Soc Psychiatry Psychiatr Epidemiol. 2012;47(9):1409-17.

8. Lenzenweger MF, Lane MC, Loranger AW, Kessler RC. DSM-IV personality disorders in the National Comorbidity Survey Replication. Biol Psychiatry. 2007;62(6):553-64.

9. Huang B, Grant BF, Dawson DA, Stinson FS, Chou SP, Saha TD, Goldstein RB, Smith SM, Ruan WJ, Pickering RP. Race-ethnicity and the prevalence and cooccurrence of diagnostic and statistical manual of mental disorders, fourth edition, alcohol and drug use disorders and Axis I and II disorders: United States, 2001 to 2002. Compr Psychiatry. 2006;47(4):252-7.

10. Ronningstam E, Baskin-Sommers AR. Fear and decision-making in narcissistic personality disorder-a link between psychoanalysis and neuroscience. Dialogues Clin Neurosci. 2013;15(2):191-201.

11. Diamond D, Meehan KB. Attachment and object relations in patients with narcissistic personality disorder: implications for therapeutic process and outcome. J Clin Psychol. 2013;69(11):1148-59.

12. Zhang T, Li H, Tang Y, Li H, Zheng L, Guo Q, Zhao S, Zhuo K, Qian Z, Wang $\mathrm{L}$, et al. Screening schizotypal personality disorder for detection of clinical high risk of psychosis in Chinese mental health services. Psychiatry Res. 2015;228(3):664-70.

13. Zhang T, Chow A, Wang L, Dai Y, Xiao Z. Role of childhood traumatic experience in personality disorders in China. Compr Psychiatry. 2012;53(6): 829-36.

14. Zhang T, Good MJ, Good BJ, Chow A, Wang L, Dai Y, Xiao Z. Age and remission of personality pathology in the psychotic disorders compared to mood and/or anxiety disorders. Int J Psychiatry Med. 2012;44(3):241-55.

15. Zhu S, Zhang T, Yang C, Wei Y, Xu L, Wang J, Chow A, Liu X, Jiang K, Xiao Z, et al. A compromise solution between overlapping and overlooking DSM personality disorders in Chinese psychiatric practice. Soc Psychiatry Psychiatr Epidemiol. 2018;53(1):99-106

16. Yang $Y$, Shen D, Wang J, Yang J. The reliability and validity of PDQ-4+ in China. Chin J Clin Psychol. 2002;10:165-8.

17. Dai Y, Xiao Z, Wang Z, Zhang $H$, Chen J. The reliability and validity of a Chinese version of the structured clinical interview for DSM-IV personality disorders. Shanghai ArchPsychiatry. 2006;18:1-4.

18. Jablensky A. The classification of personality disorders: critical review and need for rethinking. Psychopathology. 2002;35(2-3):112-6.

19. Levy KN. Subtypes, dimensions, levels, and mental states in narcissism and narcissistic personality disorder. J Clin Psychol. 2012;68(8):886-97.

20. Samuels J, Eaton WW, Bienvenu OJ 3rd, Brown CH, Costa PT Jr, Nestadt G. Prevalence and correlates of personality disorders in a community sample. Br J Psychiatry. 2002;180:536-42.

21. Torgersen S, Kringlen E, Cramer V. The prevalence of personality disorders in a community sample. Arch Gen Psychiatry. 2001;58(6):590-6.

22. Zimmerman M, Rothschild L, Chelminski I. The prevalence of DSM-IV personality disorders in psychiatric outpatients. Am J Psychiatry. 2005; 162(10):1911-8.

23. Zimmerman M, Galione JN, Chelminski I, Young D, Dalrymple K, Morgan TA. Does the diagnosis of multiple Axis II disorders have clinical significance? Ann Clin Psychiatry. 2012;24(3):195-201.

24. Ryder AG, Sunohara M, Kirmayer LJ. Culture and personality disorder: from a fragmented literature to a contextually grounded alternative. Curr Opin Psychiatry. 2015;28(1):40-5.

25. American Psychiatric Association. Diagnostic and statistical manual of mental disorders. 4th ed. Washington: American Psychiatric Association; 1994.

26. Morey LC. Personality disorders in DSM-III and DSM-III-R: convergence, coverage, and internal consistency. Am J Psychiatry. 1988;145(5):573-7. 